\title{
The loci controlling plasticity in flax
}

\author{
This article was published in the following Dove Press journal: \\ Research and Reports in Biology \\ 31 January 2012 \\ Number of times this article has been viewed
}

\author{
Cory L Bickel \\ Marshall Lukacs \\ Christopher A Cullis \\ Case Western Reserve University, \\ Cleveland $\mathrm{OH}$, USA
}

Correspondence: Christopher A Cullis Case Western Reserve University, 10900 Euclid Ave, DeGrace Hall Room 203, Cleveland OH, 44I06, USA

$\mathrm{Tel}+\mathrm{I} 2163683557$

Fax +I 2163684672

Email cac5@case.edu
Abstract: Flax undergoes heritable genomic changes in response to nutrient stress, including changes in total DNA content, rDNA copy number variation, and the appearance of Linum Insertion Sequence 1 (LIS-1). The nature of the genomic changes suggests a very different mechanism, which is not yet understood, from that of other DNA changes in response to stress, such as the activation of transposable elements. To identify the genes that control genomic changes in response to stress in flax, reciprocal crosses were made between a responsive flax line, Stormont cirrus, and an unresponsive line, Bethune. The ability of the F2 generation (from selfed F1 plants) to respond to nutrient stress was assayed using the insertion of LIS-1 as the criteria for responsiveness. Twenty-nine out of 89 F2s responded at 5 weeks, suggesting that 3-4 dominant loci were all necessary for early LIS-1 insertion. Seventy out of 76 responded at 10 weeks, indicating two dominant loci independently capable of initiating LIS-1 insertion under prolonged nutrient stress. F1 plants and their progeny with either Pl or Bethune as the maternal parent were capable of responding with LIS-1 insertion, indicating that LIS-1 insertion is under nuclear genetic control and does not involve maternal factors. Thus, a small number of loci within the genome of Stormont cirrus appear to control the ability to respond to nutrient stress with LIS-1 insertion. A genetic map of the flax genome is currently under construction, and will be used to identify these loci within the genome.

Keywords: nutrient stress, genomic plasticity, flax, Linum usitatissimum, LIS-1

\section{Introduction}

Flax is an economically important crop grown for both its fibers and its oil. Inbred lines exist that are optimized for either fiber quality (flax lines) or oil content and quality (linseed lines). One major difference between flax and linseed lines is the ability of many flax lines to respond to nutrient stress with heritable genomic alterations, while linseed lines possess stable genomes. ${ }^{1}$ The genomic alterations that take place include changes in total DNA content, copy number variation of repetitive regions, such as the ribosomal RNA genes (rDNA) and satellite regions, and the appearance of Linum Insertion Sequence 1 (LIS-1). These genomic changes occur within one generation in flax plants growing under nutrient stress conditions and can be inherited by future generations. Some moderate nutrient stress conditions give rise to plants that retain genomic plasticity, while more extreme stress conditions cause the genomic changes to be stable under further changes in nutrient regimes. The flax lines Stormont cirrus (called "plastic" or Pl) and Lyral prince are capable of giving rise to these stable lines, termed genotrophs, while other flax lines remain plastic regardless of the severity of nutrient stress. Phenotypic changes accompanying these genomic changes include submit your manuscript $\mid$ www. dovepress.com

Dovepress

http://dx.doi.org/1 0.2147/RRB.S27198
Research and Reports in Biology 2012:3 I-II

(C) 2012 Bickel et al, publisher and licensee Dove Medical Press Ltd. This is an Open Access article which permits unrestricted noncommercial use, provided the original work is properly cited. 
plant height, weight, and branching, isozyme mobility, and capsule septa hair number. ${ }^{1-5}$

Similar responses to stresses and environmental stimuli have been documented in other plant species. Amplification of satellite DNA was demonstrated in orchid in response to hormones, ${ }^{6}$ in Vicia faba during de-differentiation of root cells, ${ }^{7}$ and in tobacco in response to wounding or tobacco mosaic virus infection. ${ }^{8}$ Changes in rDNA copy number were seen in Brassica napus and Descurainia sophia during low temperature acclimation, ${ }^{9}$ and variability in DNA content influenced by light quality and quantity was demonstrated in sunflowers. ${ }^{10,11}$ Therefore, the understanding of the control of the genomic changes that take place in flax has the potential to be applied to similar changes that take place in a wide variety of plants.

The stable genotrophs derived from the Pl line have been the subjects of most studies on the genomic changes that take place in flax. A summary of the phenotypic and genotypic changes in the stable genotrophs $\mathrm{L}$ and $\mathrm{S}$ is given in Figure 1. Treatment with low or imbalanced nutrients gives rise to the small, or S, genotroph. The $\mathrm{S}$ genotroph is shorter than the original P1 line with little branching, hairy capsule septa, and altered isozyme mobility. $\mathrm{S}$ has a reduced amount of nuclear DNA and rDNA and contains LIS-1. A high nutrient treatment gives rise to the large, or $\mathrm{L}$, genotroph. $\mathrm{L}$ is much taller than S and slightly shorter than $\mathrm{Pl}$, and has significantly more branching than $\mathrm{Pl}$ or S. L has hairless capsule septa and altered isozyme mobility relative to both $\mathrm{Pl}$ and $\mathrm{S}$. L has a greater amount of nuclear DNA than Pl, similar rDNA content, and does not contain LIS-1.,12-18 Both L and S have been shown to be better adapted to the nutrient environment in which they were induced than the original Pl line, where L grows larger and produces more seeds in the high nutrient environment than

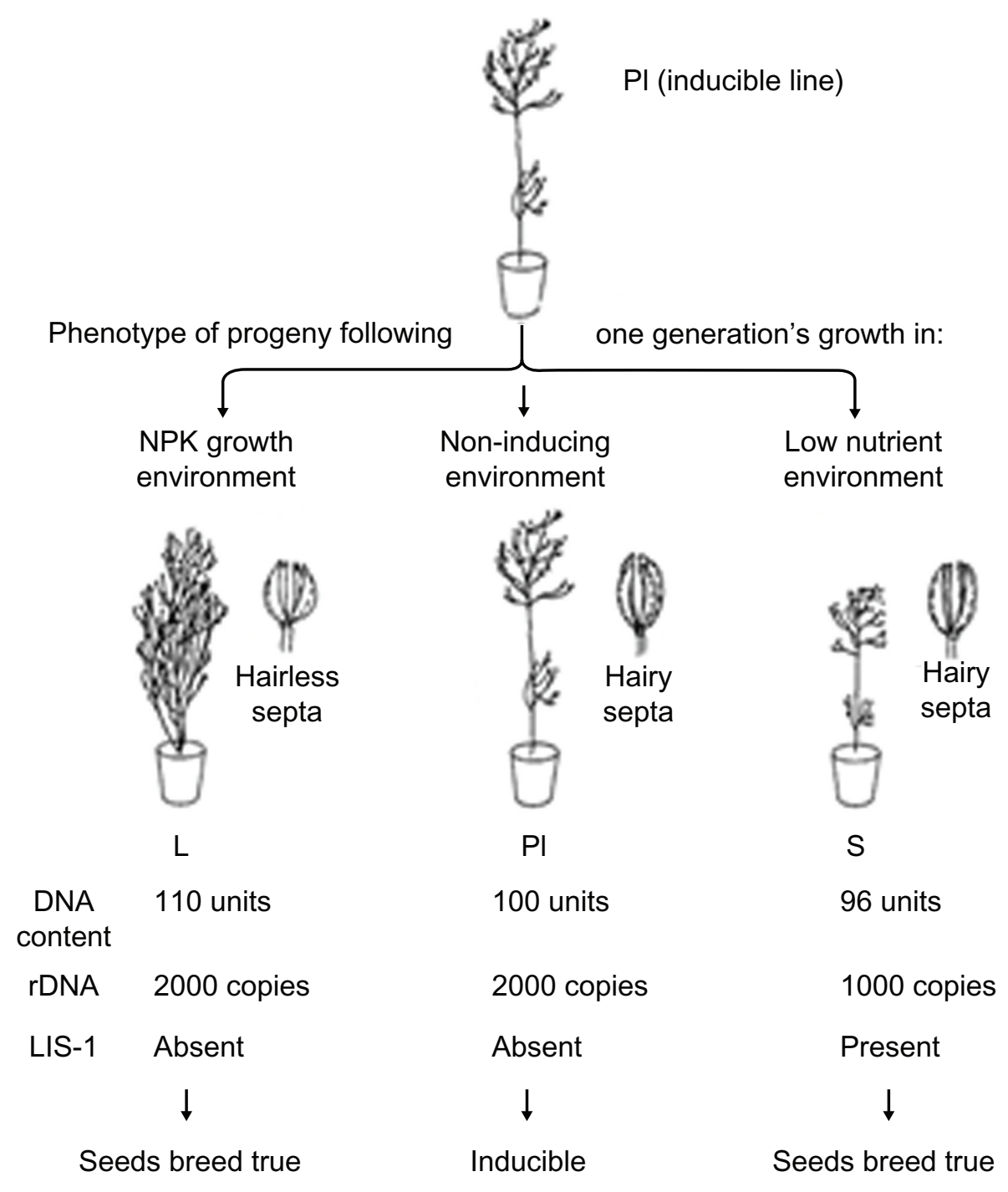

Figure I Diagram of response to nutrient stress in the Stormont cirrus (PI) flax line and characteristics of resultant genotrophs.

(C) Copyright 1983 from Molecular Biology of the Cell by Alberts et al. Reproduced by permission of Garland Science/Taylor \& Francis Books, LLC. 
$\mathrm{Pl}$ or $\mathrm{S}$, and $\mathrm{S}$ produces more seeds and exhibits less lodging than Pl or L under low nutrient conditions, although it does not grow as large (CA Cullis, unpublished data). ${ }^{19}$

The best characterized genomic change in flax is the appearance of LIS- $1 .{ }^{17}$ LIS-1 is a $5.7 \mathrm{~kb}$ sequence that is inserted into a specific, single copy target sequence (Figure 2) in the Pl genome during growth under low nutrient inducing conditions. Pl becomes homozygous for LIS-1 in the inducing generation, and LIS-1 is a stable part of the S genome in subsequent generations. Both LIS-1 and its target site have been sequenced. LIS-1 contains several short matches to the Linum EST database, and several informatically identified putative miRNAs (Pers comm, T Moss, 2011) but no large, open reading frames or homology to transposons or other mobile elements. The target sequence contains two genes on either side of the insertion site, inhibitor of growth 1, and kip-related cyclin-dependent kinase inhibitor 2. The target sequence is also highly modified when LIS-1 is inserted. A total of 129 single nucleotide polymorphisms and indels were found in the target sequence in comparisons between lines with and without LIS- $1 .{ }^{17}$ The regions that remain unaltered in the target sequence coincide roughly with the coding regions of the two genes. A TCC duplication occurs at either end of the insertion site when LIS-1 is inserted, which is similar to the footprint of the insertion of transposable elements, but, unlike transposable elements, there are no direct or inverted repeats at the ends of LIS-1. Overall, the structure of LIS- 1 is very different from that of known transposable elements, which indicates that its insertion is accomplished by a different process than the "cut and paste" or "copy and paste" mechanisms of most transposons.

The origin of LIS-1 in the genome is still uncertain. So far, the data indicate that LIS-1 is assembled or rearranged from short sequences found scattered throughout the genome. Absence of an intact LIS-1 in Pl before induction has been demonstrated by both Southern blotting and polymerase chain reaction (PCR), ${ }^{17}$ and by informatic analysis of the genomic sequence of the flax line Bethune. ${ }^{20}$ BLAST analysis of LIS-1 against the genome showed short, yet significant regions of high similarity and longer regions of lower similarity to LIS-1. The total of all matching sequences did not cover all of the LIS-1 sequence, and none of the matches were $100 \%$ identical (M Cullis, unpublished data). Because Bethune is not a responsive variety, it is possible that regions related to LIS-1 are missing from its genome that may be found in the genome of $\mathrm{Pl}$, and this is the reason that Bethune cannot insert LIS-1. However, the presence of short matches to LIS-1 scattered throughout the Bethune genome lends support to the idea that LIS-1 is assembled from these short pieces before being inserted into its target sequence.

The original measure of the responsiveness of flax to nutrient stress was plant weight at maturity, ${ }^{1}$ and measures of response since then have included changes in total DNA content using Feulgen staining ${ }^{5}$ and changes in rDNA copy number using Southern blotting and quantitative PCR. ${ }^{18,21}$ However, the most consistent and easily scorable measure of responsiveness is the insertion of LIS-1. LIS-1 insertion has

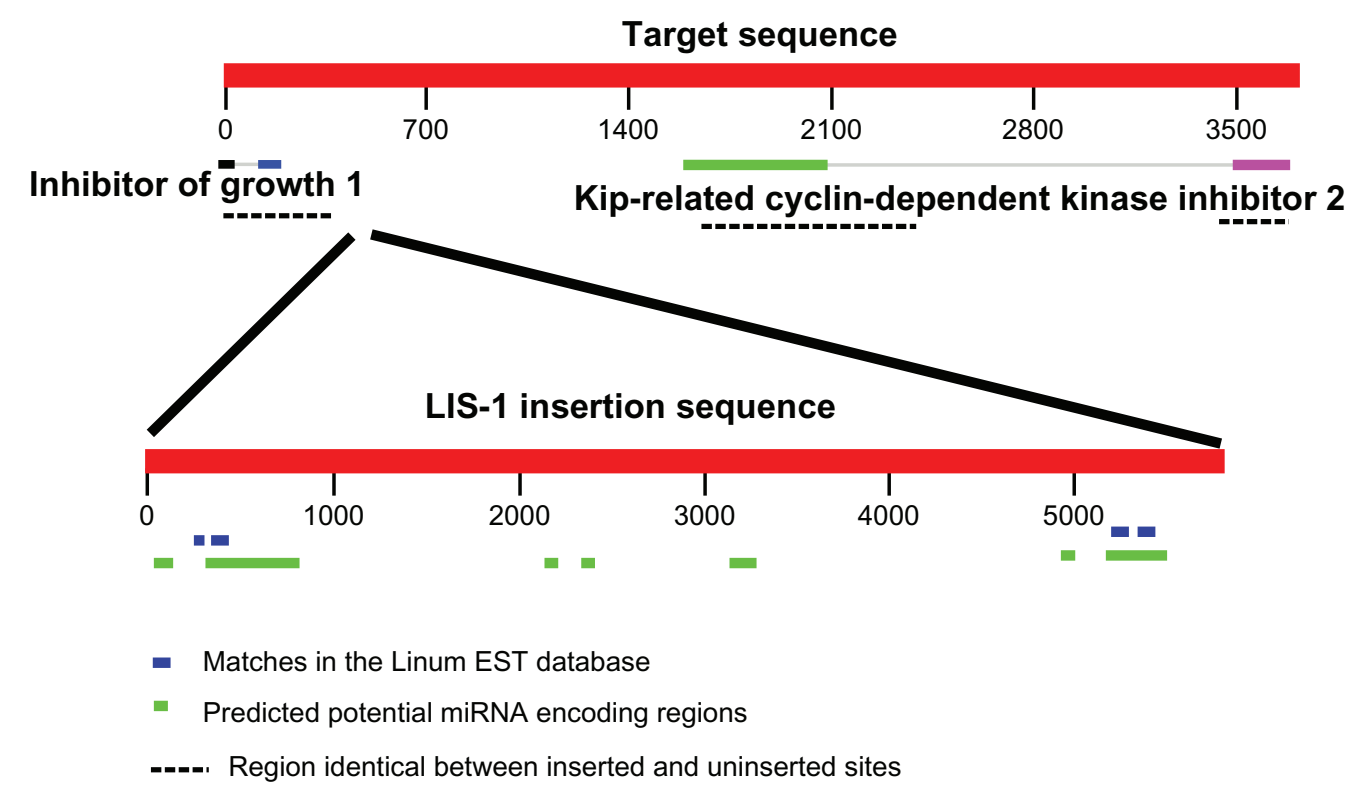

Figure 2 LIS-I and its target sequence. Two genes in the target sequence, matches in the Linum EST database, putative miRNAs, and sites within the target sequence that are unaltered during LIS-I insertion are shown (CA Cullis, unpublished data).

Abbreviation: LIS-I, Linum Insertion Sequence I. 
been demonstrated in many separate experiments in response to low nutrient inducing conditions. ${ }^{17,18}$ Its presence or absence can be easily demonstrated by PCR using primers for either the entire inserted sequence, the junctions of LIS-1 and the target sequence, or the target sequence without LIS-1. In this study the amplification of the $5^{\prime}$ junction fragment (left-hand junction in Figure 2) and the loss of amplification of the target sequence were used as determinants of responsiveness.

The lack of homology to transposable elements in LIS-1 and the lack of the intact LIS-1 sequence in Pl plants before nutrient stress indicate that the mechanism for LIS-1 insertion is different from any known mechanisms for DNA alterations in response to stress. Whether the mechanism that controls LIS-1 insertion is the same as that which controls the other genomic changes is not yet known. However, the ability to assay for LIS-1 insertion using PCR and the lack of LIS-1 seen in unstressed plants make LIS-1 an optimal marker for stress responsiveness. LIS-1 insertion was therefore chosen as the initial aspect of genomic changes for study.

To begin to understand the mechanism that guides LIS-1 assembly and insertion, the loci controlling LIS-1 insertion were analyzed in crosses between responsive and unresponsive flax lines. By examining the ability of the F1 and F2 offspring to insert LIS-1, the nature of the control of LIS-1 insertion could be determined, including the number of loci involved in controlling insertion and whether maternal or paternal factors were involved. Reciprocal crosses between the responsive $\mathrm{Pl}$ line and the unresponsive Bethune line were made, and the F1 generation was self-fertilized to generate F2 plants. Leaves collected at 5 and 10 weeks from F2 plants grown under low nutrient-inducing conditions were assayed for LIS-1. The number of plants responding at each time period can be used to determine the probable number of loci involved in controlling LIS-1 insertion. The number of plants responding at 5 weeks was consistent with 3-4 dominant loci, all of which were necessary for LIS-1 insertion, while the number responding at 10 weeks was consistent with two dominant loci, each independently capable of inducing LIS-1 insertion. The ability of F1s and F2s with both P1 and Bethune as maternal parents to insert LIS-1 demonstrated that no maternal or paternal factors were involved, and that LIS-1 insertion is under nuclear genetic control.

\section{Methods}

\section{Plant material}

All plants were watered twice daily for 15 minutes with an automatic watering system and were grown under a 16:8-hour light:dark cycle. The parent lines for the crosses,
$\mathrm{Pl}$ and Bethune, were grown under noninducing (control) conditions, which consist of $100 \mathrm{~mL}$ of a $1 \mathrm{tsp} /$ gallon Miracle-Gro ${ }^{\circledR}$ (24 N-8P-16 K; Scotts Miracle-Gro Company, Marysville, $\mathrm{OH}$ ) solution applied once a week. Reciprocal crosses were made by removing the petals and stamens from a flower on the "female" parent before the stamens had developed far enough to allow for self-pollination. Pollen from the "male" parent was applied to the stigma and seeds were collected after the pods had fully developed and dried.

Eighteen F1 plants were grown under low nutrient $\left(\mathrm{H}_{2} \mathrm{O}\right)$ - inducing conditions, which consisted of water only with no fertilizer added. Six leaves were collected at 4, 10, and 21 weeks to test for the presence of LIS-1. A total of $100 \mathrm{~F} 1$ plants were grown under noninducing conditions and allowed to self-pollinate to generate the F2 generation. Leaves were collected at 12 weeks, after flowering, to test for LIS-1. Ten seeds each from 10 of the F1 parents that consisted of five plants with $\mathrm{Pl}$ as the maternal parent and five with Bethune as the maternal parent were used in the F2 generation. A total of $100 \mathrm{~F} 2$ plants were grown under low-nutrient conditions to determine whether they were able to respond to nutrient stress. The plants were divided among ten pots with ten plants per pot, with one plant from every F1 parent per pot, so that the offspring of any F1 parent were not all inadvertently subjected to excess stress from lack of water or light. Six leaves were collected from the apex after 5 and 10 weeks for use in PCR. Twenty F2s were grown under noninducing conditions to provide a control set and allowed to self-pollinate to produce seeds for the F3 generation.

\section{DNA extraction}

Six leaves from each plant were used for DNA extraction. Leaves were put in $400 \mu \mathrm{L}$ buffer AP1 from a DNeasy Plant Minikit (Qiagen, Valencia, CA) with sterile sand and ground until no tissue clumps were visible. DNA extraction was then carried out following the manufacturer's instructions.

\section{PCR with LIS-I and target primers}

PCR was performed using $1 \mathrm{X}$ Go $\mathrm{Taq}^{\circledR}$ Green Master Mix (Promega, Madison, WI) with $100 \mathrm{nmol}$ of each primer and $1 \mu \mathrm{L}$ DNA per reaction. Primers Target L (CCCCCTTCTTCAGT TCTGCT) and 3' (GAGGATGGAAGATGAAGAAGG) were used to amplify the left hand junction of LIS-1 and its target sequence to demonstrate its insertion. Primers Target $\mathrm{L}$ and Target R (GGCTAGGGTTAGGGTTTCCA) were used to amplify the uninserted target sequence. Figure 3 shows the location of the primers within LIS-1 and the target sequence. PCR involved an initial hold of $27^{\circ} \mathrm{C}$, then 2 minutes at $95^{\circ} \mathrm{C}$, 


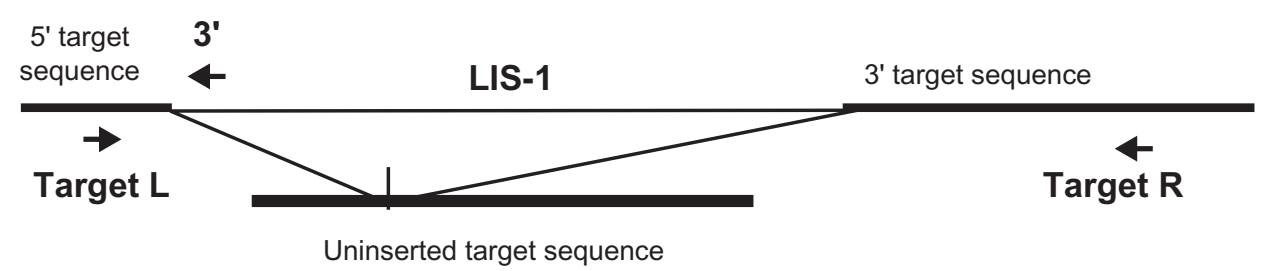

Figure 3 Structure of the target sequence with and without LIS-I insertion and location of primers in LIS-I and the target sequence. Primers Target L and $3^{\prime}$ amplify the junction of LIS-I and the target sequence when LIS-I is present. Primers Target $L$ and Target $R$ amplify the uninserted target sequence. Target L, Target R, and $3^{\prime}$ refer to primer names only, and $3^{\prime}$ reflects the orientation of the primer sequence rather than the orientation of LIS-I, which lies in the same $5^{\prime} \rightarrow 3^{\prime}$ direction as the target sequence.

Abbreviation: LIS-I, Linum Insertion Sequence I.

followed by 35 cycles of $94^{\circ} \mathrm{C}$ for 30 seconds, $55^{\circ} \mathrm{C}$ for 30 seconds, and $72^{\circ} \mathrm{C}$ for 1 minute. A final extension step at $72^{\circ} \mathrm{C}$ for 5 minutes was followed by an indefinite hold at $4^{\circ} \mathrm{C}$. PCR products were separated on $1.5 \%(\mathrm{w} / \mathrm{v})$ agarose gels in $0.5 \mathrm{X}$ TBE $(1 \mathrm{X}$ TBE $=100 \mathrm{mM}$ Tris, $90 \mathrm{mM}$ boric acid, $1 \mathrm{mM}$ EDTA, $\mathrm{pH}$ 8.4). F2s were scored as responsive if they contained the $250 \mathrm{bp}$ band that represents the LIS-1 target sequence junction, and unresponsive if they did not.

\section{Sequencing of amplified bands}

Bands with the expected size for the PCR product containing the LIS-1 and target sequence junction were excised from the gel and DNA was extracted using a Qiagen gel extraction kit. DNA was re-amplified using Go Taq ${ }^{\circledR}$ Green Master Mix using the conditions described above. PCR products $(5 \mu \mathrm{L})$ were run on agarose gels $1.5 \%(\mathrm{w} / \mathrm{v})$ for quantification with Easy Ladder I (Bioline, Tauton, MA, USA) as a reference. The remaining PCR products were purified using a Qiagen PCR cleanup kit and sequenced by MWG Operon (Huntsville, AL). The resultant sequences were compared with LIS-1 and target sequences using BLAST software (NIH, Bethesda, MD) to confirm the presence of the junction site. Several other bands of different sizes were present, and were extracted and sequenced in the same way. These were compared to the LIS-1 and target sequences and to the nucleotide database using BLAST, and to the flax genome database $^{21}$ using the site's BLAST function.

\section{Results}

\section{Responsiveness of FI generation under control and inducing conditions}

F1 plants were grown under control conditions and allowed to self-pollinate to produce the F2 generation of plants. All ten $\mathrm{F} 1$ parents of the F2s used in this study were tested for the presence of the LIS-1 and target sequence junction site. None of the F1 parents had amplification with the Target L and 3' primers that amplify the LIS-1 and target sequence junction (Figure 4), demonstrating that LIS-1 was absent from this generation of plants, and that the F2s that contained LIS-1, described below, did so because its insertion was induced by nutrient stress rather than inherited from the F1 parent. All the individuals did amplify the expected band with the primer pair Target $\mathrm{L}$ and Target $\mathrm{R}$ confirming the presence of an unaltered target site (data not shown).

A total of $18 \mathrm{~F} 1$ plants were grown under $\mathrm{H}_{2} \mathrm{O}$-inducing conditions to determine if this generation was able to respond to nutrient stress. All but one plant responded to the nutrient stress with the insertion of the expected $250 \mathrm{bp}$ band (Figure 5, top). The lack of amplification seen with plant 12 may be due to low DNA in this sample, as there was also no amplification seen with primers Target $\mathrm{L}$ and Target $\mathrm{R}$, which should amplify if LIS-1 is not inserted. The fact that the majority of plants responded demonstrates that the genes that convey responsiveness to stress are dominant, and that there are genes present in $\mathrm{Pl}$ that convey responsiveness in this line, rather than genes in Bethune that prevent responsiveness.

The Target $\mathrm{L}$ and Target $\mathrm{R}$ primers were used to determine whether the plants still had alleles in which LIS-1 was not inserted, where the $5^{\prime}$ and $3^{\prime}$ target sequences were still adjacent to each other. In the F1 plants for which P1 was the maternal parent, four-fifths still had the 1400 bp band that

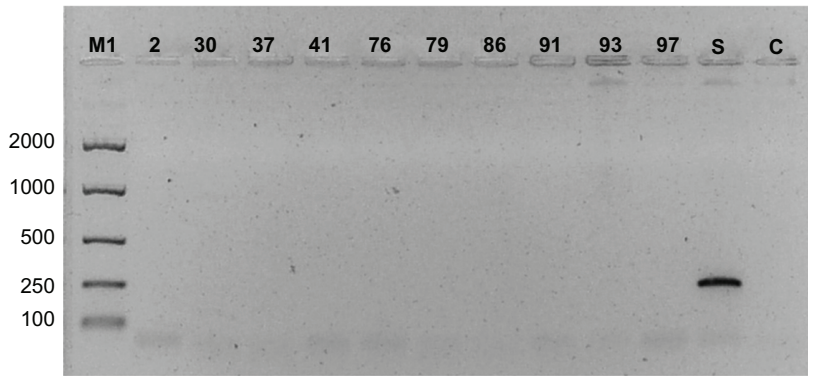

Figure 4 PCR products from FI parent DNA with Target $L$ and $3^{\prime}$ primers. The 250 bp band in $S$ was the expected size for the LIS-I and target sequence junction fragment. None of the FI parents had this band, demonstrating the absence of LIS-I in these plants, and confirming that the F2s that contain LIS-I did not inherit it from the $\mathrm{FI}$ parents, but rather inserted it in response to stress.

Abbreviations: MI, Bioline Easy Ladder I; 2-97, FI parents; S, small genotroph with LIS-I insertion used as positive control; C, no-DNA control, PCR, polymerase chain reaction; LIS-I, Linum Insertion Sequence I. 


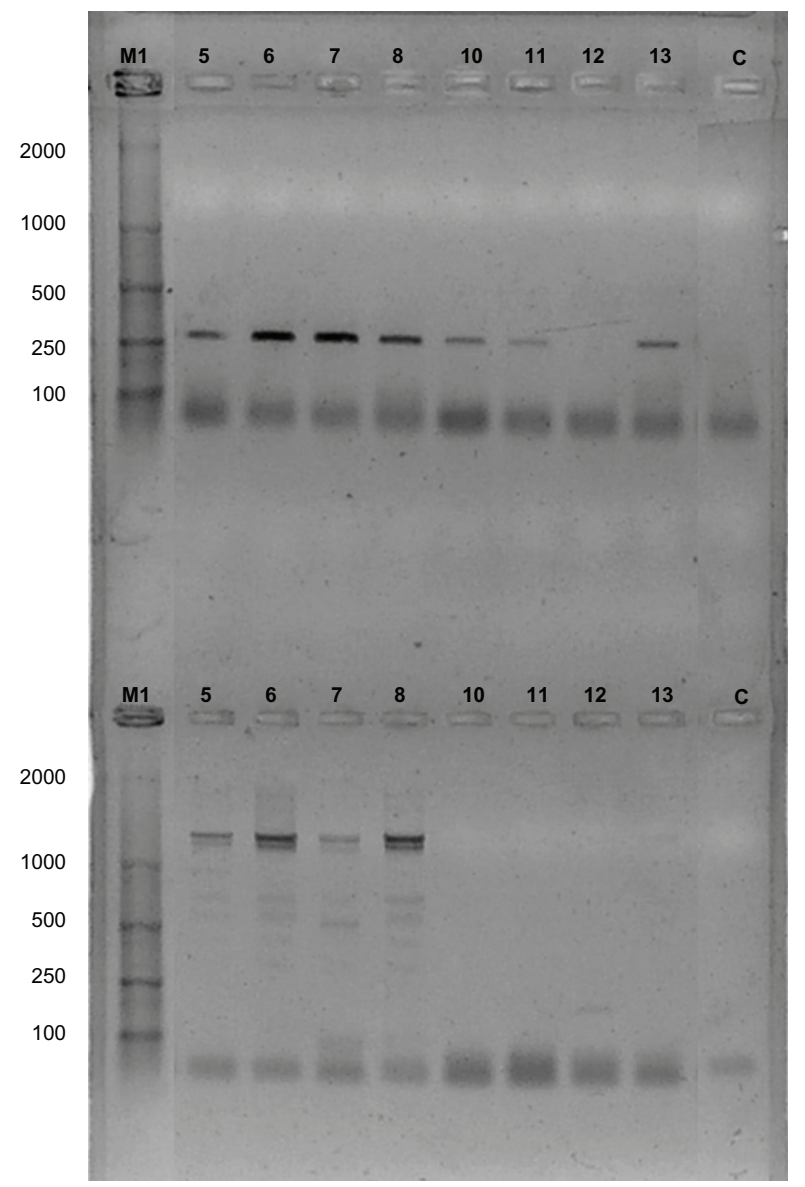

Figure $5 \mathrm{FI}$ plants grown under inducing conditions for 21 weeks amplified with primer pairs Target $L$ and $3^{\prime}$, Target $L$ and Target R. The 250 bp LIS-I junction band (top) amplifies in Fls with both Stormont cirrus (PI) and Bethune as the maternal parent. This indicates that the formation of the junction fragment is not dependent on maternal factors. The $1400 \mathrm{bp}$ band (bottom) that represents the target site without LIS-I insertion is only present in FIs that have $\mathrm{PI}$ as the maternal parent, indicating that maternal factors from PI may play a role in the ability of these plants to become homozygous for LIS-I or in the timing of its insertion.

Abbreviations: MI, Bioline Easy Ladder I; 5-13 FI plants; 5-10, PI $\times$ Bethune; II-13, Bethune $\times \mathrm{PI}$; S, positive control; C, no-DNA control; LIS-I, Linum Insertion Sequence I.

represents the uninserted target sequence (Figure 5, lanes 5-10, bottom). In plants with Bethune as the maternal parent, one plant had no band, one had only a very faint band, and one was inconclusive because of the lack of amplification with any primers (Figure 5, lanes 11-13, bottom). It appears that plants with Bethune as the maternal parent become homozygous for LIS-1 earlier than those with Pl as the maternal parent.

The fact that the appearance of LIS-1 does not depend on the direction of the cross suggests that the ability to insert LIS-1 is not dependent on maternal factors. However, the fact that fewer plants with $\mathrm{Pl}$ as the maternal parent become homozygous for LIS-1 suggests that the maternal factors play some role in creating homozygosity. The uninserted target site may be seen in these plants because one allele of the target sequence prohibits LIS-1 insertion, or because the DNA extracted from these leaves comes from a mixture of cells in which some have become homozygous and some have not. Maternal factors from Pl may play a role in either the accessibility of the target sequence for insertion or the timing of the insertion processes.

These F1 plants did not respond by inserting LIS-1 until the third leaf collection at 21 weeks. DNAs from previous collections at 4 and 10 weeks showed no LIS- 1 insertion. The timeline for LIS-1 insertion appears to be much longer than that seen in $\mathrm{Pl}$, which begins to respond at 3 weeks. ${ }^{18}$ This is likely due to the heterozygosity of the F1s for responsiveness genes, which necessitates a more prolonged stress for activation of the stress response.

\section{Responsiveness of F2s: LIS-I insertion}

$\mathrm{F} 2$ offspring were grown under $\mathrm{H}_{2} \mathrm{O}$ inducing conditions and leaves were collected at 5 and 10 weeks to test for stress responsiveness. DNA was extracted from the leaves and was amplified using primers Target L and $3^{\prime}$. The presence of LIS-1 was determined by the appearance of a $250 \mathrm{bp}$ band that represented the junction site of LIS- 1 and the $5^{\prime}$ target sequence (Figure 6, band 1), which was confirmed by sequencing, described below.

\section{Frequency of LIS-I insertion in F2s}

At 5 weeks, 29 plants had inserted LIS-1, while 60 had not (Table 1). These numbers are not significantly different from those expected for a ratio of 27:37 ratio expected for three dominant necessary genes $\left(x^{2}=3.37, P>0.05\right)$ nor from an $81: 175$ ratio for four dominant genes $\left(x^{2}=0.036, P>0.8\right)$, all necessary for induction of LIS-1 insertion in response to stress. Previous studies on the timing of LIS-1 insertion have shown that the $5^{\prime}$ end of LIS- 1 can be detected as early as

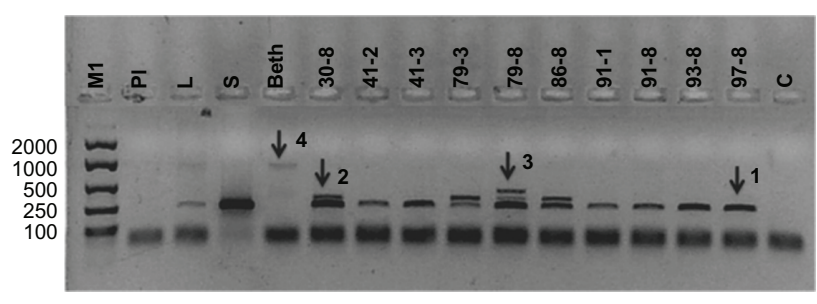

Figure 6 F2 DNA amplified with primers Target $L$ and $3^{\prime}$. Band I was confirmed as the LIS-I and target sequence junction fragment. Bands 2, 3, and 4 were unexpected and were also sequences and analyzed. Bands 2 and 3 appear to be transition products in the assembly of LIS-I-like labile genomic regions, while Band 4 represents product from a weak priming site for primer $3^{\prime}$ in the target sequence.

Abbreviations: MI, Bioline Easy Ladder I; PI and Bethune, F0 parents; L, large genotroph; S, small genotroph, positive control; 30-n, 4I-n, 79-n, 86-n, 9I-n, 93-n, 97-n, F2s; C, no-DNA control; I-4, bands extracted for sequencing. 
Table I LIS-I insertion in F2 plants grown under inducing conditions at 5 and 10 weeks

\begin{tabular}{llll}
\hline Growth period & LIS-I + & LIS-I - & \% Responding \\
\hline 5 weeks & 29 & 60 & 33 \\
I0 weeks & 70 & 6 & 92 \\
\hline
\end{tabular}

3 weeks, and that LIS- 1 is fully inserted and homozygous by 7 weeks. ${ }^{18}$ Therefore, the 5 -week collection may have been too early for all plants capable of responding to nutrient stress to do so. It is also possible that because not all plants in the F2 generation will be homozygous for the necessary loci, as the original Pl plants are, response time could be delayed relative to the response time seen in $\mathrm{Pl}$. This is consistent with the late responsiveness seen in the $\mathrm{F} 1$ generation, which contains only one copy of each Pl gene, and does not respond until after 10 weeks of growth under low-nutrient conditions.

As seen in the F1 generation, LIS-1 was inserted in F2s with both $\mathrm{Pl}$ and Bethune as the maternal parent. LIS-1 appearance at 5 weeks was seen in offspring from each of the ten F1 parent plants. Also, LIS-1 insertion was seen in plants from all of the ten pots in which the F2s were grown, with the exception of pot ten. The number of LIS-1+ plants per pot was not significantly different from those expected from a random distribution among the pots $\left(x^{2}=12.72\right.$, $P>0.1$ ). This eliminates possible effects on LIS-1 insertion from water levels and amount of light that may have varied among pots.

At 10 weeks, 70 plants contained LIS- 1 while six did not (Table 1). These numbers are not significantly different from a ratio of 15:1 $\left(x^{2}=0.34, P>0.5\right)$ that would be expected if two independent dominant loci control the ability to respond to stress. The numbers are significantly different $\left(x^{2}=11.85, P<0.001\right)$ from the expected ratio of $3: 1$ for a single dominant gene. As seen in the 5 -week data, there was no correlation between responsiveness and the F1 parent, or with pot number $\left(x^{2}=14, P>0.1\right)$.

No F2s were fully homozygous for LIS- 1 at either time point, as demonstrated by amplification with primers Target $\mathrm{L}$ and Target $\mathrm{R}$ of the $1400 \mathrm{bp}$ band that represents the uninserted target sequence. Whether this is due to a delay in the full insertion of LIS-1 similar to that seen in the F1s, or to a lack of genes necessary for stabilizing the insertion in the target sequence is not clear at this point. The 5-week and 10 -week data for LIS-1 insertion indicate different numbers of loci involved in the stress response. The 5-week data suggest that there are 3-4 dominant loci that are all necessary to initiate the earliest insertion of LIS-1. These loci may be activated by low levels of short-term stress and would result in early LIS-1 insertion in the relatively few F2 plants where all loci were present. The two loci indicated by the 10 -week data may represent "emergency" or "late" loci, each of which can act independently to initiate the stress response, specifically LIS-1 insertion, under conditions of severe or prolonged stress.

\section{Characterization of bands amplified with Target $L$ and 3'}

Amplification with primers Target $L$ and $3^{\prime}$ yielded four sizes of bands with some of the F2 DNAs (Figure 6). Band 1 was approximately $250 \mathrm{bp}$, which was the expected size for the junction site of LIS- 1 and its target sequence. Also present was a $350 \mathrm{bp}$ band (2), a $400 \mathrm{bp}$ band (3), and a 1000 bp band (4), which was present in the Bethune parent as well as in some F2s. The number of F2s that had band 1 at the 5- and 10-week collections is discussed in detail above. Band 2 was present in four F2s in samples from the 5-week collection, and ten in the 10-week collection. Also, in some F2s, band 2 is seen in the 5 -week collection but not at 10 weeks, indicating that the sequence is not a stable part of the genome in these plants. Likewise, band 3 appeared in only one F2 plant at 5 weeks, and in five other plants at 10 weeks, but was no longer seen in the first plant by this time. Bands 2 and 3 are not seen in the $\mathrm{Pl}$ or Bethune parents, or in S, in which LIS-1 is stably inserted. Together these data indicate that bands 2 and 3 may represent transitional sequences in the assembly of LIS- 1 or of other yet uncharacterized insertion elements. Band 4 amplified in $20 \mathrm{~F} 2 \mathrm{~s}$ at 5 weeks and in $18 \mathrm{~F} 2 \mathrm{~s}$ at 10 weeks. The amplification intensity varied significantly among the F2s in which it did amplify, with some showing very faint bands as seen in Bethune in Figure 6, and some giving much more intense bands.

All of these bands were sequenced and analyzed using BLAST to confirm that band 1 was the LIS- 1 target sequence junction, and to characterize the other bands (see Supplementary data). Band 1 aligned with the $5^{\prime}$ target sequence as well as the $5^{\prime}$ end of LIS- 1 as expected, which confirms that this band does represent the LIS-1 and target sequence junction in the F2s. While differences of three base pairs were seen between this band and both the target and LIS-1 sequences, the precise alignment of most of the sequence demonstrates the consistency and reproducibility of LIS-1 assembly and insertion.

Analysis of bands 2 and 3 further confirms that they may represent transitional sequences. Only short matches to LIS-1 are seen in these bands, indicating that they are transitional forms of other insertion sequences. These transitional bands 
may arise because of the presence of the Bethune genome in these crosses. It appears that when the flax genome is heterozygous, new labile sequences are created. These sequences are ephemeral, however, and are not selected for in the meristem and stably incorporated into the final genomic configuration.

The sequence data for band 4 showed that it was amplified from a weak priming site for primer $3^{\prime}$ within the target sequence. Its variation in intensity may be due to differences in amplification conditions among PCR runs, or it may be that F2s that amplify with differing intensities have alterations in the target sequence at this site that affect the binding of primer $3^{\prime}$. The $3^{\prime}$ end of band 4 lies in a region of the target sequence that is altered upon LIS-1 insertion. Differences in the timing of the alterations among the F2s may explain the variation in priming and amplification efficiency.

\section{Discussion}

The F1 parents grown under control conditions did not contain LIS-1, demonstrating that any appearance of LIS-1 in the F2 generation was not due to inheritance from the previous generation. This is further supported by the large number of F2 plants that were negative for LIS-1 at 5 weeks but positive by 10 weeks. The appearance of LIS-1 in many plants was demonstrated to occur during growth of the F2 generation, and is not the result of F1 outcrossing with LIS-1 containing plants. Genomic changes occurring during the growth of one generation of flax have been previously demonstrated in traits such as total DNA content, rDNA copy number changes, and LIS-1 insertion, ${ }^{18,22-24}$ so the fact that these genomic changes are induced by stress rather than due to outcrossing or contamination has also previously been well established in many separate experiments.

The ability of Pl flax plants to insert LIS-1 in response to low-nutrient stress conditions has been previously demonstrated, ${ }^{17,18}$ but the control of its insertion has not been understood. Here it is shown that the gene(s) controlling LIS-1 insertion are dominant, demonstrated by the fact that the majority of F1 plants grown under inducing conditions were positive for LIS-1. The F1s resulting from reciprocal crosses between $\mathrm{Pl}$ and Bethune were capable of inserting LIS-1, which ruled out the involvement of maternal factors and demonstrated that LIS-1 insertion is under nuclear genetic control. However, the ability of plants to become homozygous for LIS-1 was affected by the maternal parent, where plants with $\mathrm{Pl}$ as the maternal parent had not become homozygous for LIS-1 by 21 weeks. This is surprising, given that $\mathrm{Pl}$ is the responsive parent, and it would be expected that factors from Pl would facilitate rather than limit the ability to become homozygous. One possibility is that factors from $\mathrm{Pl}$ are copy-number dependent and so in the heterozygous state delay the progression to homozygosity for LIS-1, rather than alter the ability to become homozygous.

Possible copy-number dependence was also demonstrated for LIS-1 insertion. The long delay in LIS-1 insertion seen in the F1 plants relative to Pl plants that are homozygous for the genes controlling insertion ${ }^{18}$ may indicate that the number of alleles inherited from Pl determine the timing of LIS-1 insertion. This is supported by the data from the F2s, where the majority of plants did not respond until 10 weeks' growth in stress conditions. The number of F2 plants responding at 5 weeks is not significantly different from the one-quarter expected to respond if only plants that were homozygous for one of the Pl alleles were capable of a response to nutrient stress in the usual time frame $\left(x_{2}=2.73, P>0.1\right)$. The much higher number seen responding at 10 weeks may indicate that heterozygous plants need more prolonged levels of stress to activate responsiveness genes or take longer to build up critical levels of some yet unidentified "response factor." Once the specific genes for LIS-1 insertion are identified, the role of homozygosity in the timing of the response will be easy to study using simple PCR or sequencing at these loci.

The number of loci controlling LIS-1 insertion has also previously been unknown. The data for responsiveness of the F2 plants has indicated for the first time the involvement of several loci in this process. The number of F2 plants responding with LIS-1 insertion at 5 weeks is consistent with 3-4 loci which are all necessary for responsiveness, while the number responding at 10 weeks indicates that two dominant loci are independently capable of initiating LIS-1 insertion. The response of F2s with both $\mathrm{Pl}$ and Bethune as the maternal parent further demonstrate that LIS-1 insertion is under nuclear genetic control rather than maternal factors. The different number of loci indicated at the two time points may be due to a delay in response due to heterozygosity for Pl-derived alleles, or may reflect two sets of control loci, where the 3-4 "early" loci work together to respond to low levels of stress, while the two "late" loci can independently initiate LIS-1 insertion in response to prolonged or severe stress.

Informatic analysis of the genomic sequence of the unresponsive Bethune line had shown that the complete LIS-1 sequence was not present within the genome (M Cullis, unpublished data). It has been suggested that the 
lack of LIS-1 progenitor sequences is the reason that Bethune is unable to respond to stress with LIS-1 insertion. However, the small number of loci indicated by the data presented here precludes the involvement of sequences from the Bethune genome in limiting the ability to insert LIS-1. Because the Bethune genome does not contain the entire LIS-1 sequence, it could potentially limit the ability to insert LIS-1 to only those F2s that had inherited all LIS-1 progenitor sequences from Pl. If this were the case, all responding plants would have to inherit both the progenitor sequences and the genes controlling LIS-1 insertion. The very high number of plants responding at 10 weeks rules out this scenario, unless the LIS-1 progenitor sequences are clustered together and very tightly linked to each of the two dominant loci that appear to control insertion, but previous data have also shown that neither complete LIS-1 nor large fragments of LIS-1 are present in Pl. Comparison of LIS-1 and the Bethune genome using BLAST shows that the LIS-1 progenitor sequences that are present in Bethune are widely scattered throughout the genome. Unless the organization of the Pl genome is vastly different than that of Bethune, clustering and linkage of progenitor sequences and control genes is unlikely. It appears that only the inheritance of the genes controlling LIS-1 insertion is necessary and that differences in LIS-1 progenitor sequences in the Bethune genome do not affect the assembly and insertion of LIS-1.

The data presented here clarify several aspects of the control of LIS-1 insertion in response to nutrient stress. The gene(s) controlling insertion have been shown to be dominant, and insertion to be under nuclear genetic control. The numbers of loci involved in early and late LIS-1 insertion have been identified, and are low enough to rule out the involvement of Bethune genomic sequences in constraining LIS-1 insertion. A genetic mapping project using the Bethune genome is currently underway with the aim of identifying the loci that control LIS-1 insertion. Once these loci have been placed on the map, the specific genes that control LIS-1 insertion can be identified, and the nature of these genes can shed light on the mechanism by which LIS-1 is assembled and inserted into the target sequence. This map can also be used to identify loci that control other genomic changes in flax, which will indicate whether all changes are controlled by "master switch" genes, or whether each aspect of the stress response in flax is controlled and inherited separately. A better comprehension of the mechanisms that control genomic changes in flax will aid in understanding the processes that generate genetic variability in plants, identifying potentially useful stress resistance traits for crop plants, and understanding how plants maintain stable or plastic genomes in the face of environmental challenges.

\section{Acknowledgments}

This research was supported by the GAANN fellowship, number P200 A090191 to CLB. ML was partially supported by a received a SPUR Fellowship. Use of greenhouse facilities at the Case Western Reserve University Squire Valleevue Farm was also supported by the Olgebay Fund.

\section{Disclosure}

The authors report no conflict of interest in this work.

\section{References}

1. Durrant A. The environmental induction of heritable change in Linum. Heredity. 1962;17:27-61.

2. Cullis CA. Quantitative variation in the ribosomal RNA genes in flax genotrophs. Heredity. 1979a;42(2):237-246.

3. Cullis CA. Segregation of the isozymes of flax genotrophs. Biochem Genet. 1979b;17(3/4):391-401.

4. Durrant A, Nicholas DB. An unstable gene in flax. Heredity. 1970;25: 513-527.

5. Evans GM. Nuclear changes in flax. Heredity. 1968;23:25-38.

6. Nagl W, Rucker W. Effect of phytohormones on thermal denaturation profiles of Cymbidium DNA: indication of differential DNA replication. Nuc Acid Res. 1976;3:2033-2039.

7. Natali L, Cavallini A, Cremonini R, Bassi P, Cionini PG. Amplification of nuclear DNA sequences during induced plant cell dedifferentiation. Cell Diff. 1986;18:157-161.

8. Gense MT. DNA synthesis in wounded or TMV-infected leaves from sensitive and hypersensitive tobacco plants. Cell Diff. 1980;9 117-124.

9. Laroche A, Geng XM, Singh J. Differentiation of freezing tolerance and vernalization responses in Cruciferae exposed to a low temperature. Plant Cell Envir. 1992;15:439-445.

10. Natali L, Cavallini A, Cionini G, Sassoli O, Cionini PG, Durante M Nuclear DNA changes within Helianthus annus L.: Changes within single progenies and their relationships with plant development. Theor Appl Genet. 1993;85:506-512.

11. Johnston JS, Jensen A, Czeschin DG, Price HJ. Environmentally induced nuclear 2C DNA content instability in Helianthus annus (Asteraceae). Am J Bot. 1996;83:1113-1120.

12. Durrant A. Induction and growth of flax genotrophs. Heredity. 1971;27: 277-298.

13. Tyson H, Fieldes MA. Molecular-weight and net charge of peroxidase isozymes in F1 hybrids between L and S flax genotrophs. Biochem Genet. 1982;20:919-927.

14. Cullis CA. Molecular aspects of the environmental induction of heritable changes in flax. Heredity. 1977;38:129-154.

15. Cullis CA. Environmentally induced DNA changes in plants. $C R C$ Critical Rev Plant Sci. 1983;1:117-129.

16. Cullis CA. Phenotypic consequences of environmentally induced changes in plant DNA. Trends Gene. 1986;2:307-310.

17. Chen Y, Schneeberger RG, Cullis CA. A site-specific insertion sequence in flax genotrophs induced by environment. New Phytol. 2005;167: 171-180.

18. Chen Y, Lowenfeld R, Cullis CA. An environmentally induced adaptive (?) insertion event in flax. Int J Genet Mol Biol. 2009;1(3):38-47.

19. Johnson C, Moss T, Cullis CA. Environmentally induced heritable changes in flax. JVis Exp. 2011;47:pii:2332. 
20. linum.ca [homepage on the Internet]. Edmonton, AB: University of Alberta; c2011 [updated November 4, 2011]. Available at: http://carpel.ccis.ualberta.ca/. Accessed November 9, 2011.

21. Cullis CA. Mechanisms and control of rapid genomic changes in flax. Ann Bot. 2005;95:201-206.

22. Cullis CA. Environmentally induced changes in ribosomal RNA cistron number in flax. Heredity. 1976;36(1):73-79.
23. Evans GM, Durrant A, Rees H. Associated nuclear changes in the induction of flax genotrophs. Nature. 1966;212:697-699.

24. Cullis CA, Charlton LM. The induction of ribosomal DNA changes in flax. Plant Sci Lett. 1981;20:213-217. 


\section{Supplementary data}

Sequence data for bands I-4

Band I

TTTAGGNACTCTGGNNNNCTGAAATTGGGGATTGAAAGGGAGGTCTTGGAGGAGTCCGGATCTGGAGC CAGGAAATCAAATTCTGGATCTGATTCGGAATGGCACAACCCATCTGAATCGTCCAAAAAATGGTACCCTT GATCTAACGCCTGAAATTTTGGGGGCATGTAAGTAGTCTCCTTCCTTCTTCATCTTCCATCC

\section{Band 2}

CNNNTNTTCGATCTTGCTNNNNNNNTTCAGTTCTNCTTNNTGGTTGNTAAACCNACTCCATCTCTGCTCG GAGCCACAAGTGATCCCATAACCTTTGTCATGTAATTTTCCGAGATCTTTGGTTTCTTCTATGACAGCGACGACA GCTCGAACTTCAGTGTCAGGCTTTGGAGGATCCTTTCCACGATCTTCTTCTCGTCGATAATCTCTCCAG GACTTTGATCTGGTTCACTATCTCTGCAACACGAGAATGAAAGTCTTTGATGCTTTCGCCTTCTTCATCTT CATCCT

\section{Band 3}

ACTTGATGAGGTGTTATAGTAATCAACCACCTTCATGTCTCCCTACCTGAGGCTAATAAACTCACCCCTCTTG TTCTTCTGCCACTGCTCTGGCATGAACTCGTTCTTGAAAATGACTAAGACTTCCTCCCAATCCCTTCTGAAAT GGTCATACTCAACCTCTAACTAGTTGCTCTACCACTCCTTTCTTGCACCTCTCAACATGNAGGCAGCATACA ACACTCTCTACTCTGGCGCACCTCTCAGATACATGAACACGTTGGTCAGTCTCTTAGCAAATCAACTATTAA CCGAGGATCTGTGGTCTCCCCATCAAAAGTAATGGCGCCCACTCGAATCAGCTCCTTGAAATCTAGCCTATC CACAGGCGGATCCTTCTTCATCTTCCATCC

\section{Band 4}

AGGNACTCTGNGCGTATCTGNAATTGGGGATTGAAAGGGAGGTCTTGGAGAAACCCCGTTTTGTTCCNT GGAATCGGTGGTTGGTTCAGAGGAGCAATGGTAATCCCCATCNGAGGGTACCGGGATGGAACCAAAT TNNCTANCGAAATANAANTTGGGGGANGTGGTTAATAACCGCCCTTCTGGTGGATGANCNTNGNTATGC CATAACCATCCCCGAACCTCCTCGTTGCCAAGTCTAAATCCAAGCTGTTACAAGATAAACTTTGTATTATTTTCA CACCATATAATATACTACATACATTATTATAAAAAAAAGTTATTTATGAGGGCAATATAATACTTTAACTTTTATT TAATCAAATATAACTCTTTATTTTGAGTTAATATAGCAGAATAATTTTTATAAAATCCAAAATATTTTATAAGTA TCACCTTTTCAAATAATATATTATTTTATTTTAAAATACTACATATTACAAAATAACTCATAAAACACATCAAAAT TAATCTCATTGAACTAAAAATTAAAAAAAACAAATAAAATTTGCGAAGGAAACATGATTTAAAGTTTAAAC TGTAGAGACATGATCACAAAATCTAAAGAAAAAATAAAATTAAGAAGAGAGAAAAATGAAAGAAAAATA TCACAGAAAAAGAAAGAAGAGCGAGTAGGAAACACGTGCGGCTGTGGGCCTCCGTAGCCCGTCGAATCAA TGGCTGAGATTTAGTTTCAGTGGTTGTTTGGGATTCGAAATCCGACGCAGCTTCCCGTTTCCAATTTCAGTT TTACAAATACGAAGACTTTCGTCCCCCGTTTCCCATTTCTCTCCTTCTTCATCNNNCCATCC

Publish your work in this journal

Research and Reports in Biology is an international, peer-reviewed, open access journal publishing original research, reports, editorials, reviews and commentaries on all areas of biology including animal biology, biochemical biology, cell biology, ecological studies, evolutionary biology, molecular biology, plant science and botany. The manuscript management system is completely online and includes a very quick and fair peer-review system. Visit http://www.dovepress. $\mathrm{com} /$ testimonials.php to read real quotes from published authors. 